\title{
Approach to Fabricate Rigid Substrate for 2.4 GHz Inverted-F Antenna Using a Room Temperature Curable Dielectric Ink on Photo and Nanopaper
}

\author{
A. K. Sowpati ${ }^{1)}$, M. Nelo ${ }^{1)}$, J. Varghese ${ }^{1)}$, H. Liimatainen ${ }^{2)}$, M. Visanko ${ }^{2)}$, M. T. \\ Sebastian ${ }^{1)}$ and H . Jantunen ${ }^{1)}$ \\ 1) Microelectronics and Materials Physics Laboratories, Faculty of Information \\ Technology and Electrical Engineering, University of Oulu, P.O. Box 8000, FI- \\ 90014, Finland. \\ 2) Fiber and Particle Engineering Unit, Faculty of Technology, University of Oulu, \\ P.O. Box 8000, FI-90014, Finland. \\ Corresponding Author:
}
A.K.Sowpati
e-mail: arun@ee.oulu.fi

\begin{abstract}
The effect of a room temperature curable dielectric ink $\left(\mathrm{ZrSiO}_{4}\right)$ printed on commercial photo paper and prepared nanopaper on the dielectric properties at $2.4 \mathrm{GHz}$ are studied. In both cases, the dielectric layer decreased the relative permittivity and dielectric loss and made the flexible substrates rigid. For the nanopaper, the permittivity decreased from 4.7 to 3.57 and the loss value from 0.12 to 0.04 . The measured decreases for the photo paper were from 3.12 to 2.61 and from 0.09 to 0.05 , respectively. In the performance of the simulated and fabricated inverted-F antennas (IFA), the effect of the dielectric layer could be observed in the decrease of its frequency with about 130
\end{abstract}


$\mathrm{MHz}$ mainly due to the thicker substrate. The measured total efficiency and gain were $83 \%$ and $3.4 \mathrm{~dB}$. The proposed approach could be in the future used for further development of the antenna by modification of the dielectric ink with different additives.

\section{Keywords}

Room temperature curable dielectric ink; low loss tangent; Inverted F Antenna (IFA); WLAN; printing; paper; Nanocellulose

\section{Introduction}

Wireless communication wearable devices are becoming popular in many emerging applications. Modern wearable applications like smart watches use wireless technologies like Bluetooth, Wi-Fi, long-term evolution (LTE) and global positioning system (GPS), thus requiring integration of an antenna into the measuring unit. The design of antennas suitable for wrist applications is a challenging task due to the requirements of the compact and especially thin design and low manufacturing costs [1]. The inverted-F antenna (IFA) structures are widely used in wearable applications because of their simple and flexible design, low fabrication cost, and reliable performance [2,3]. On the other hand, printable electronics can produce very thin, lowcost, and lightweight structures. It is thus clear that fabrication of antennas using printable electronics would offer an optimal solution to realizing flat, inexpensive antennas.

Paper is a desired candidate for a low-cost substrate for RF printed electronic applications. It has a low surface profile and, with an appropriate coating, it is suitable 
for fast printing processes such as direct write methodologies instead of the traditional metal etching techniques. Conductive inkjet printed antennas on flexible, low-cost paper-based substrates have been reported for RFID (Radio-frequency Identification) and WSN (Wireless Sensor Network) applications [4]. For printed antennas, an interesting new option is paper based on nanocellulose (denoted here as nanopaper), which has a densely packed, layered structure allowing highly conductive and sensitive antenna lines at higher frequencies. However, the dielectric properties of common and nanopaper depend on manufacturing route and used raw materials [5-8]. Thus, the dielectric properties such as dielectric constant $\left(\varepsilon_{\mathrm{r}}\right)$ and loss tangent $(\tan \delta)$ have to be characterized before designing the high-frequency structures [5, 9-11]. However, one main disadvantage of these paper-based substrates for high-frequency antennas is their dielectric losses being $0.07-0.2$ at $2.5 \mathrm{GHz}[9,11]$.

In this paper, the feasibility of room temperature curable dielectric ink on temperature sensitive photo and nanopaper substrates. The dielectric properties of the substrates with and without dielectric coating are measured at $2.4 \mathrm{GHz}$, and the performance of the fabricated IFA are also measured. IFA designed in this paper is off ground antenna type having higher antenna efficiency compared to IFA [3] which is on ground antenna type. 


\section{Materials and methods}

\subsection{Paper substrates}

Two different substrate materials were selected with the first one being a commercially available Japanese Epson photo glossy photographic paper (denoted here as photo) having a thickness of $200 \mathrm{um}$. The measured AFM (Atom Force Microscope, Veeco Dimension 3100, US) average surface roughness (Ra) of the paper was $14 \mathrm{~nm}$. For the second, a nanopaper made of bleached hardwood kraft pulp fibers in accordance to work published earlier by Liimatainen et al. [12] was selected. The nanofibrils obtained through subsequent periodate-chlorite oxidation had an average lateral dimension of 3$5 \mathrm{~nm}$ and carboxyl content of $1.75 \mathrm{mmol} / \mathrm{g}$. A vacuum filtration method was used to prepare transparent and homogenous substrates from aqueous nanofibril suspension [13].

The fabricated cellulose nanofibrils characteristics were analyzed by a Tecnai G2 Spirit Transmission Electron Microscope (FEIEurope, Eindhoven, Netherlands). The nanofibril samples were diluted and well distributed into a $10 \mathrm{~mL}$ plastic test tube. A tiny droplet $(7 \mu \mathrm{L})$ of the dilution was dosed on the top of butvar and carbon-coated copper grid. Uranyl acetate $(2 \% \mathrm{w} / \mathrm{v})$ was applied as a negative stain to cover the samples. The excess amount of the sample and uranyl acetate was removed with a filter paper. The stained samples were dried at room temperature and later analyzed at 100 $\mathrm{kV}$ under standard conditions. Quemesa CCD camera was used to take the images. 3$5 \mathrm{~nm}$ widths of the individual nanofibrils shown in Fig. 1. (a) were measured by TEM 
image analysis software (Olympus Soft Imaging Solutions GMBH, Munster, Germany) over 100 well-distributed nanofibrils.

FESEM (Field Emission Scanning Electron Microscopy, JOEL S-4800, Hitachi, Japan) was used to study the structural properties of nanopaper substrates in the planar direction. Prior to FESEM imaging, the samples were conditioned overnight in a desiccator to remove moisture. Later coated with gold/palladium using the SEM Sputter Coater to improve the sample's electric conductivity. The images of the structure were captured from the secondary electrons released from the surface of the sample after bombardment with a beam of electrons accelerated at $1.0 \mathrm{kV}$. Fig. 1. (b) shows that the nanopaper has consisted of dense $\left(321.47 \mathrm{~g} / \mathrm{cm}^{3}\right)$ and an entangled network of individual nanofibrils without any visible larger aggregates [13]. The thickness and Ra value of the nanopaper were $30 \mathrm{um}$ and $160 \mathrm{~nm}$, respectively.

\subsection{Dielectric inks formulation and their properties}

The fabrication procedure of the dielectric $\mathrm{ZrSiO}_{4}$ ink printed on photo and nanopaper is reported earlier [14]. A homogeneous and stable suspension of $\mathrm{ZrSiO}_{4}$ was obtained with controlled particle size, and the optimized composition of the binder. The $60 \mathrm{wt}$. $\%$ amount of $\mathrm{ZrSiO}_{4}$ particles with a controlled size was mixed 35 wt.\% of the anhydrous xylene ethanol solvent system and $5 \mathrm{wt}$ \% of polyvinyl butyral binder. Fig. 2 shows the microstructure of dielectric layer on paper substrates after printing and room temperature curing. The $\mathrm{ZrSiO}_{4}$ ceramic grains were covered with a 
homogeneously dispersed polymeric binder (Fig. 2. (a). The average surface roughness obtained by the AFM measurements (Fig. 2. (b)-(c)) to the area of $10 \mu \mathrm{m} \times 10 \mu \mathrm{m}$ to the photo and nanopaper with the dielectric layer showed increased values being 540 $\mathrm{nm}$ and $330 \mathrm{~nm}$, respectively. This increase is understandable due to the large particle size distribution of the $\mathrm{ZrSiO}_{4}$ powder. Although the surface roughness plays an important role in the performance of the antenna especially effecting to the accuracy of the printed lines, still the achieve smoothness is low compared to the most common commercial PCBs. Fig. 2. (a) shows that in the printed and cured ink the $\mathrm{ZrSiO}_{4}$ ceramic grains were covered with a homogeneously dispersed polymeric binder.

\subsection{Antenna structure}

The schematic layout of the proposed printed IFA covering 2.4 -2.7 GHz (WLAN, LTE band 7) for wrist and headset applications is shown in Fig. 3. (a). The designed was done using CST Microwave studio. The whole structure included an antenna element, its ground plane and feeding, and the short. The dimensions between feed and shot were varied to minimize return loss close to $2.6 \mathrm{GHz}$.

The antenna had a uniform strip width of $1 \mathrm{~mm}$. FR4 with copper tape is used for $40 \mathrm{~mm} \times 30 \mathrm{~mm}$ ground plane. The size of substrate for the antenna element made of photo or nanopaper with and without dielectric layer on top was $6 \mathrm{~mm} \times 20 \mathrm{~mm}$. The conductive lines were screen-printed on the element with Dupont 5064H silver ink and cured at $130{ }^{\circ} \mathrm{C}$. A $50 \mathrm{ohm}$ coaxial cable was used to feed the antenna. 
Fig. 3. (b-e) shows the fabricated antennas with the copper tape and 50-ohm coaxial feeding cable. The dielectric layer clearly made especially the nanopaper more rigid thus preventing deformation of the antenna structure.

\section{Results and discussion}

The measured relative permittivity $\left(\varepsilon_{\mathrm{r}}\right)$ and dielectric loss tangent $(\tan \delta)$ values of photo and nanopaper with and without dielectric ink are shown in Table I. The printed and cured thickness of dielectric layers on photo and nanopaper substrates was $80 \mu \mathrm{m}$ and $60 \mu \mathrm{m}$, respectively. The extracted $\varepsilon_{\mathrm{r}}$ and $\tan \delta$ of the $\mathrm{ZrSiO}_{4}$ dielectric layer itself were thus 1.98 and 0.007 at $2.4 \mathrm{GHz}$. The measured $\varepsilon_{\mathrm{r}}$ values for the paper substrates correlated well with one reported earlier [4, 9, 11], especially taking into account that the permittivity of a nanopaper is reported to increase with the density [10].

The results also show that the dielectric layer brought the tan $\delta$ value to the same level (0.04-0.05 at $2.4 \mathrm{GHz})$ decreasing it significantly especially in the case of nanopaper. Furthermore, the $\varepsilon_{\mathrm{r}}$ values decreased. The decrease of both of these values is understandable due to the low permittivity and loss values of the dielectric layer.

The simulated return loss (Fig. 4. (a)) for all antennas correlate well to the measured one (Fig. 4. (b)). In all cases the printed dielectric layer decreased the frequency of the antenna about $130 \mathrm{MHz}$ although the effective permittivity decreased with the dielectric layer. The reason for this is the increased thickness of the substrates. 
The total efficiency (Fig. 5. (a)) of the antenna made on nanopaper with and without dielectric layer were found to be $83 \%$ and $79 \%$, respectively. The related value for the antenna based on photopaper were about $76 \%$ and $74 \%$. Thus the $\mathrm{ZrSiO}_{4}$ dielectric layer slightly improved the efficiencies due to the lower dielectric losses. The peak gain of the photo and nanopaper antennas (Fig. 5. (b)) were $3.17 \mathrm{~dB}$ and $3.4 \mathrm{~dB}$, respectively, with slight improvement with the dielectric layer. The achieve antenna performances are close to the ones reported earlier for IFA at $2.4 \mathrm{GHz}$ [15]. All these measurement result are in line with the simulations.

The results show that the introduced dielectric room temperature curable ink is feasible for the proposed antenna structure. Especially it decreased the dielectric loss of the antenna substrate when printed on photo and nanopaper. It also improved the stiffness of the antennas still enabling thin structures.

Fig. 6 shows the measured radiation pattern of the antennas of nanocellulose, photo paper substrates with and without dielectric inks. All the antennas had an omni directional radiation patterns. 
Fig. 6. Measured farfiled radiation patterns of the photo and nanopaper antennas with and without dielectric layer in (a) XZ plane and (b, c) YZ and XY planes.

\section{Conclusions}

The approach using printed room temperature curable low loss dielectric ink on antenna substrate made of photo or nanopaper is studied. The dielectric layer decreased both permittivity and dielectric loss of the photo and nanopaper from maximum of 0.12 to minimum of 0.04 at $2.4 \mathrm{GHz}$. The printed layer also made these paper-type substrates more rigid still having low thickness. In the performance of the IFA it decreased the frequency and slightly improved the radiation efficiency. The present study shows the advantage of printed room temperature curable dielectric ink, which is feasible for even temperature sensitive substrates. The proposed approach could be also used in the future to develop the antenna in many different ways. The size of the antenna could be e.g. decreased by increasing the permittivity of the dielectric ink without loosing the good antenna performance.

\section{References}

1. C. H. Wu, K. L. Wong, Y. C. Lin and S. W. Su, Microwave Opt Lett. 49, 942 (2007).

2. P. Salonen, L. Sydanheimo, M. Keskilammi and M. Kivikoski, in Proceedings of the Third International Symposium on Wearable Computers (1999), pp. 95-98. 
3. V. K. Palukuru, A. Pekonen, V. Pynttari, R. Mäkinen, J. Hagberg and H. Jantunen, Microwave Opt Lett. 51, 12 (2009).

4. G. Shaker, A. Rida, S. Safavi-Naeini, M. M. Tentzeris and S. Nikolaou, in EUCAP proceedings (2011), pp.3001-3004.

5. S. Simula, S. Ikäläinen and K. Niskanen, J. Imag. Sci. Technol. 43, 5 (1999).

6. M. Nogi, N. Komoda, K. Otsuka and K. Suganuma, Nanoscale, 4395 (2013).

7. J. Huang, Y. Zhou, L. Dong, Z. Zhou and R. Liu, Composites Science and Technology 138, 40 (2017).

8. D. L. Bras, M. Strømme and A. Mihranyan, The Journal of Physical Chemistry B, 5911 (2015).

9. L. Yang, A. Rida, R. Vyas and M. Tentzeris, IEEE Transactions on Microwave Theory and Techniques, Vol. 55, no.12, 2894 (2007).

10. T. Inui, H. Koga, M. Nogi, N. Komoda and K. Suganuma, Adv. Mater. 27, 1112 (2015).

11. H. Yagyu, S. Ifuku and M. Nogi, Flex. Print, Electron. 2, 014003 (2017).

12. H. Liimatainen, M. Visanko, J. A. Sirviö, O. E. O. Hormi and J. Niinimäki, Biomacromolecules 13, 1592 (2012).

13. H. Liimatainen, N. Ezekiel, R. Sliz, K. Ohenoja, J. A. Sirviö, L. Berglund, O. Hormi and J. Niinimäki, ACS Applied Materials and Interfaces 5, 13412 (2013).

14. J. Varghese, M. Teirikangas, J. Puustinen, H. Jantunen and M. T. Sebastian, Journal of Materials Chemistry C, 9240 (2015).

15. D. E. Anagnostou, A. A. Gheethan, A. K. Amert and K. W. Whites, Journal of display technology 6, 11 (2010). 


\title{
Approach to Fabricate Rigid Substrate for 2.4 GHz Inverted-F Antenna Using a Room Temperature Curable Dielectric Ink on Photo and Nanopaper
}
A. K. Sowpati ${ }^{1)}$, M. Nelo ${ }^{1)}$, J. Varghese ${ }^{1)}$, H. Liimatainen ${ }^{2)}$, M. Visanko ${ }^{2)}$, M. T.
Sebastian ${ }^{1)}$ and H . Jantunen ${ }^{1)}$
1) Microelectronics and Materials Physics Laboratories, Faculty of Information Technology and Electrical Engineering, University of Oulu, P.O. Box 8000, FI- 90014, Finland.
2) Fiber and Particle Engineering Unit, Faculty of Technology, University of Oulu, P.O. Box 8000, FI-90014, Finland.
Corresponding Author:
A.K.Sowpati
e-mail: arun@ee.oulu.fi

\begin{abstract}
The effect of a room temperature curable dielectric ink $\left(\mathrm{ZrSiO}_{4}\right)$ printed on commercial photo paper and prepared nanopaper on the dielectric properties at $2.4 \mathrm{GHz}$ are studied. In both cases, the dielectric layer decreased the relative permittivity and dielectric loss and made the flexible substrates rigid. For the nanopaper, the permittivity decreased from 4.7 to 3.57 and the loss value from 0.12 to 0.04 . The measured decreases for the photo paper were from 3.12 to 2.61 and from 0.09 to 0.05 , respectively. In the performance of the simulated and fabricated inverted-F antennas (IFA), the effect of the dielectric layer could be observed in the decrease of its frequency with about 130
\end{abstract}


$\mathrm{MHz}$ mainly due to the thicker substrate. The measured total efficiency and gain were $83 \%$ and $3.4 \mathrm{~dB}$. The proposed approach could be in the future used for further development of the antenna by modification of the dielectric ink with different additives.

\section{Keywords}

Room temperature curable dielectric ink; low loss tangent; Inverted F Antenna (IFA); WLAN; printing; paper; Nanocellulose

\section{Introduction}

Wireless communication wearable devices are becoming popular in many emerging applications. Modern wearable applications like smart watches use wireless technologies like Bluetooth, Wi-Fi, long-term evolution (LTE) and global positioning system (GPS), thus requiring integration of an antenna into the measuring unit. The design of antennas suitable for wrist applications is a challenging task due to the requirements of the compact and especially thin design and low manufacturing costs [1]. The inverted-F antenna (IFA) structures are widely used in wearable applications because of their simple and flexible design, low fabrication cost, and reliable performance $[2,3]$. On the other hand, printable electronics can produce very thin, lowcost, and lightweight structures. It is thus clear that fabrication of antennas using printable electronics would offer an optimal solution to realizing flat, inexpensive antennas.

Paper is a desired candidate for a low-cost substrate for RF printed electronic applications. It has a low surface profile and, with an appropriate coating, it is suitable 
for fast printing processes such as direct write methodologies instead of the traditional metal etching techniques. Conductive inkjet printed antennas on flexible, low-cost paper-based substrates have been reported for RFID (Radio-frequency Identification) and WSN (Wireless Sensor Network) applications [4]. For printed antennas, an interesting new option is paper based on nanocellulose (denoted here as nanopaper), which has a densely packed, layered structure allowing highly conductive and sensitive antenna lines at higher frequencies. However, the dielectric properties of common and nanopaper depend on manufacturing route and used raw materials [5-8]. Thus, the dielectric properties such as dielectric constant $\left(\varepsilon_{\mathrm{r}}\right)$ and loss tangent $(\tan \delta)$ have to be characterized before designing the high-frequency structures [5, 9-11]. However, one main disadvantage of these paper-based substrates for high-frequency antennas is their dielectric losses being $0.07-0.2$ at $2.5 \mathrm{GHz}[9,11]$.

In this paper, the feasibility of room temperature curable dielectric ink on temperature sensitive photo and nanopaper substrates. The dielectric properties of the substrates with and without dielectric coating are measured at $2.4 \mathrm{GHz}$, and the performance of the fabricated IFA are also measured. IFA designed in this paper is off ground antenna type having higher antenna efficiency compared to IFA [3] which is on ground antenna type. 


\section{Materials and methods}

\subsection{Paper substrates}

Two different substrate materials were selected with the first one being a commercially available Japanese Epson photo glossy photographic paper (denoted here as photo) having a thickness of $200 \mathrm{um}$. The measured AFM (Atom Force Microscope, Veeco Dimension 3100, US) average surface roughness (Ra) of the paper was $14 \mathrm{~nm}$. For the second, a nanopaper made of bleached hardwood kraft pulp fibers in accordance to work published earlier by Liimatainen et al. [12] was selected. The nanofibrils obtained through subsequent periodate-chlorite oxidation had an average lateral dimension of 3$5 \mathrm{~nm}$ and carboxyl content of $1.75 \mathrm{mmol} / \mathrm{g}$. A vacuum filtration method was used to prepare transparent and homogenous substrates from aqueous nanofibril suspension [13].

The fabricated cellulose nanofibrils characteristics were analyzed by a Tecnai G2 Spirit Transmission Electron Microscope (FEIEurope, Eindhoven, Netherlands). The nanofibril samples were diluted and well distributed into a $10 \mathrm{~mL}$ plastic test tube. A tiny droplet $(7 \mu \mathrm{L})$ of the dilution was dosed on the top of butvar and carbon-coated copper grid. Uranyl acetate $(2 \% \mathrm{w} / \mathrm{v})$ was applied as a negative stain to cover the samples. The excess amount of the sample and uranyl acetate was removed with a filter paper. The stained samples were dried at room temperature and later analyzed at 100 $\mathrm{kV}$ under standard conditions. Quemesa CCD camera was used to take the images. 3$5 \mathrm{~nm}$ widths of the individual nanofibrils shown in Fig. 1. (a) were measured by TEM 
image analysis software (Olympus Soft Imaging Solutions GMBH, Munster, Germany) over 100 well-distributed nanofibrils.

FESEM (Field Emission Scanning Electron Microscopy, JOEL S-4800, Hitachi, Japan) was used to study the structural properties of nanopaper substrates in the planar direction. Prior to FESEM imaging, the samples were conditioned overnight in a desiccator to remove moisture. Later coated with gold/palladium using the SEM Sputter Coater to improve the sample's electric conductivity. The images of the structure were captured from the secondary electrons released from the surface of the sample after bombardment with a beam of electrons accelerated at $1.0 \mathrm{kV}$. Fig. 1. (b) shows that the nanopaper has consisted of dense $\left(321.47 \mathrm{~g} / \mathrm{cm}^{3}\right)$ and an entangled network of individual nanofibrils without any visible larger aggregates [13]. The thickness and Ra value of the nanopaper were $30 \mathrm{um}$ and $160 \mathrm{~nm}$, respectively.

\subsection{Dielectric inks formulation and their properties}

The fabrication procedure of the dielectric $\mathrm{ZrSiO}_{4}$ ink printed on photo and nanopaper is reported earlier [14]. A homogeneous and stable suspension of $\mathrm{ZrSiO}_{4}$ was obtained with controlled particle size, and the optimized composition of the binder. The $60 \mathrm{wt}$. $\%$ amount of $\mathrm{ZrSiO}_{4}$ particles with a controlled size was mixed 35 wt.\% of the anhydrous xylene ethanol solvent system and $5 \mathrm{wt}$ \% of polyvinyl butyral binder. Fig. 2 shows the microstructure of dielectric layer on paper substrates after printing and room temperature curing. The $\mathrm{ZrSiO}_{4}$ ceramic grains were covered with a 
homogeneously dispersed polymeric binder (Fig. 2. (a). The average surface roughness obtained by the AFM measurements (Fig. 2. (b)-(c)) to the area of $10 \mu \mathrm{m} \times 10 \mu \mathrm{m}$ to the photo and nanopaper with the dielectric layer showed increased values being 540 $\mathrm{nm}$ and $330 \mathrm{~nm}$, respectively. This increase is understandable due to the large particle size distribution of the $\mathrm{ZrSiO}_{4}$ powder. Although the surface roughness plays an important role in the performance of the antenna especially effecting to the accuracy of the printed lines, still the achieve smoothness is low compared to the most common commercial PCBs. Fig. 2. (a) shows that in the printed and cured ink the $\mathrm{ZrSiO}_{4}$ ceramic grains were covered with a homogeneously dispersed polymeric binder.

\subsection{Antenna structure}

The schematic layout of the proposed printed IFA covering 2.4 -2.7 GHz (WLAN, LTE band 7) for wrist and headset applications is shown in Fig. 3. (a). The designed was done using CST Microwave studio. The whole structure included an antenna element, its ground plane and feeding, and the short. The dimensions between feed and shot were varied to minimize return loss close to $2.6 \mathrm{GHz}$.

The antenna had a uniform strip width of $1 \mathrm{~mm}$. FR4 with copper tape is used for $40 \mathrm{~mm} \times 30 \mathrm{~mm}$ ground plane. The size of substrate for the antenna element made of photo or nanopaper with and without dielectric layer on top was $6 \mathrm{~mm}$ x $20 \mathrm{~mm}$. The conductive lines were screen-printed on the element with Dupont 5064H silver ink and cured at $130{ }^{\circ} \mathrm{C}$. A $50 \mathrm{ohm}$ coaxial cable was used to feed the antenna. 
Fig. 3. (b-e) shows the fabricated antennas with the copper tape and 50-ohm coaxial feeding cable. The dielectric layer clearly made especially the nanopaper more rigid thus preventing deformation of the antenna structure.

\section{Results and discussion}

The measured relative permittivity $\left(\varepsilon_{\mathrm{r}}\right)$ and dielectric loss tangent $(\tan \delta)$ values of photo and nanopaper with and without dielectric ink are shown in Table I. The printed and cured thickness of dielectric layers on photo and nanopaper substrates was $80 \mu \mathrm{m}$ and $60 \mu \mathrm{m}$, respectively. The extracted $\varepsilon_{\mathrm{r}}$ and $\tan \delta$ of the $\mathrm{ZrSiO}_{4}$ dielectric layer itself were thus 1.98 and 0.007 at $2.4 \mathrm{GHz}$. The measured $\varepsilon_{\mathrm{r}}$ values for the paper substrates correlated well with one reported earlier $[4,9,11]$, especially taking into account that the permittivity of a nanopaper is reported to increase with the density [10].

The results also show that the dielectric layer brought the tan $\delta$ value to the same level (0.04-0.05 at $2.4 \mathrm{GHz})$ decreasing it significantly especially in the case of nanopaper. Furthermore, the $\varepsilon_{\mathrm{r}}$ values decreased. The decrease of both of these values is understandable due to the low permittivity and loss values of the dielectric layer. The simulated return loss (Fig. 4. (a)) for all antennas correlate well to the measured one (Fig. 4. (b)). In all cases the printed dielectric layer decreased the frequency of the antenna about $130 \mathrm{MHz}$ although the effective permittivity decreased with the dielectric layer. The reason for this is the increased thickness of the substrates. 
The total efficiency (Fig. 5. (a)) of the antenna made on nanopaper with and without dielectric layer were found to be $83 \%$ and $79 \%$, respectively. The related value for the antenna based on photopaper were about $76 \%$ and $74 \%$. Thus the $\mathrm{ZrSiO}_{4}$ dielectric layer slightly improved the efficiencies due to the lower dielectric losses. The peak gain of the photo and nanopaper antennas (Fig. 5. (b)) were $3.17 \mathrm{~dB}$ and $3.4 \mathrm{~dB}$, respectively, with slight improvement with the dielectric layer. The achieve antenna performances are close to the ones reported earlier for IFA at $2.4 \mathrm{GHz}$ [15]. All these measurement result are in line with the simulations.

The results show that the introduced dielectric room temperature curable ink is feasible for the proposed antenna structure. Especially it decreased the dielectric loss of the antenna substrate when printed on photo and nanopaper. It also improved the stiffness of the antennas still enabling thin structures.

Fig. 6 shows the measured radiation pattern of the antennas of nanocellulose, photo paper substrates with and without dielectric inks. All the antennas had an omni directional radiation patterns.

\section{Conclusions}

The approach using printed room temperature curable low loss dielectric ink on antenna substrate made of photo or nanopaper is studied. The dielectric layer decreased both permittivity and dielectric loss of the photo and nanopaper from maximum of 0.12 to minimum of 0.04 at $2.4 \mathrm{GHz}$. The printed layer also made these paper-type substrates 
more rigid still having low thickness. In the performance of the IFA it decreased the frequency and slightly improved the radiation efficiency. The present study shows the advantage of printed room temperature curable dielectric ink, which is feasible for even temperature sensitive substrates. The proposed approach could be also used in the future to develop the antenna in many different ways. The size of the antenna could be e.g. decreased by increasing the permittivity of the dielectric ink without loosing the good antenna performance.

\section{References}

1. C. H. Wu, K. L. Wong, Y. C. Lin and S. W. Su, Microwave Opt Lett. 49, 942 (2007).

2. P. Salonen, L. Sydanheimo, M. Keskilammi and M. Kivikoski, in Proceedings of the Third International Symposium on Wearable Computers (1999), pp. 95-98.

3. V. K. Palukuru, A. Pekonen, V. Pynttari, R. Mäkinen, J. Hagberg and H. Jantunen, Microwave Opt Lett. 51, 12 (2009).

4. G. Shaker, A. Rida, S. Safavi-Naeini, M. M. Tentzeris and S. Nikolaou, in EUCAP proceedings (2011), pp.3001-3004.

5. S. Simula, S. Ikäläinen and K. Niskanen, J. Imag. Sci. Technol. 43, 5 (1999).

6. M. Nogi, N. Komoda, K. Otsuka and K. Suganuma, Nanoscale, 4395 (2013).

7. J. Huang, Y. Zhou, L. Dong, Z. Zhou and R. Liu, Composites Science and Technology 138, 40 (2017). 
8. D. L. Bras, M. Strømme and A. Mihranyan, The Journal of Physical Chemistry B, 5911 (2015).

9. L. Yang, A. Rida, R. Vyas and M. Tentzeris, IEEE Transactions on Microwave Theory and Techniques, Vol. 55, no.12, 2894 (2007).

10. T. Inui, H. Koga, M. Nogi, N. Komoda and K. Suganuma, Adv. Mater. 27, 1112 (2015).

11. H. Yagyu, S. Ifuku and M. Nogi, Flex. Print, Electron. 2, 014003 (2017).

12. H. Liimatainen, M. Visanko, J. A. Sirviö, O. E. O. Hormi and J. Niinimäki, Biomacromolecules 13, 1592 (2012).

13. H. Liimatainen, N. Ezekiel, R. Sliz, K. Ohenoja, J. A. Sirviö, L. Berglund, O. Hormi and J. Niinimäki, ACS Applied Materials and Interfaces 5, 13412 (2013).

14. J. Varghese, M. Teirikangas, J. Puustinen, H. Jantunen and M. T. Sebastian, Journal of Materials Chemistry C, 9240 (2015).

15. D. E. Anagnostou, A. A. Gheethan, A. K. Amert and K. W. Whites, Journal of display technology 6, 11 (2010). 
Fig. 1. (a) TEM image of nanofibrils (scale bar: $500 \mathrm{~nm}$ ) and (b) FESEM image of nanopaper substrate in planar direction (scale bar: $1 \mu \mathrm{m}$ ).

Fig. 2. (a) FESEM image of surface microstructure, and (b, c) AFM 3D surface image of cured ink on photo and nano paper.

Fig. 3. (a) Schematic layout of proposed screen printed inverted-F antenna and (b, c, d, e) antenna structures fabricated on nanopaper with the dielectric layer, nanopaper, photopaper, photopaper with the dielectric layer.

Fig. 4. (a) Simulated and (b) measured return loss of the photo and nanopaper antennas with and without dielectric layer .

Fig. 5. (a) Measured total efficiency and (b) gain of the photo and nanopaper antennas with and without the dielectric layer.

Fig. 6. Measured farfiled radiation patterns of the photo and nanopaper antennas with and without dielectric layer in (a) XZ plane and (b, c) YZ and XY planes. 


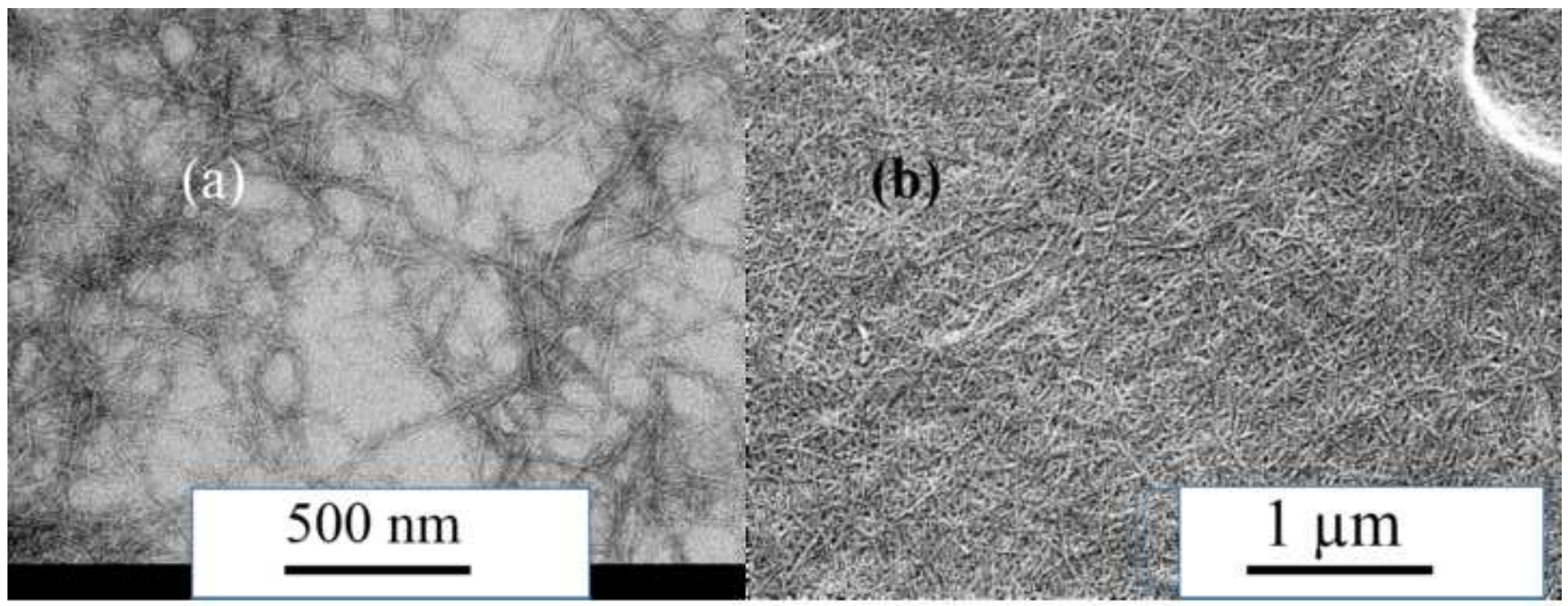



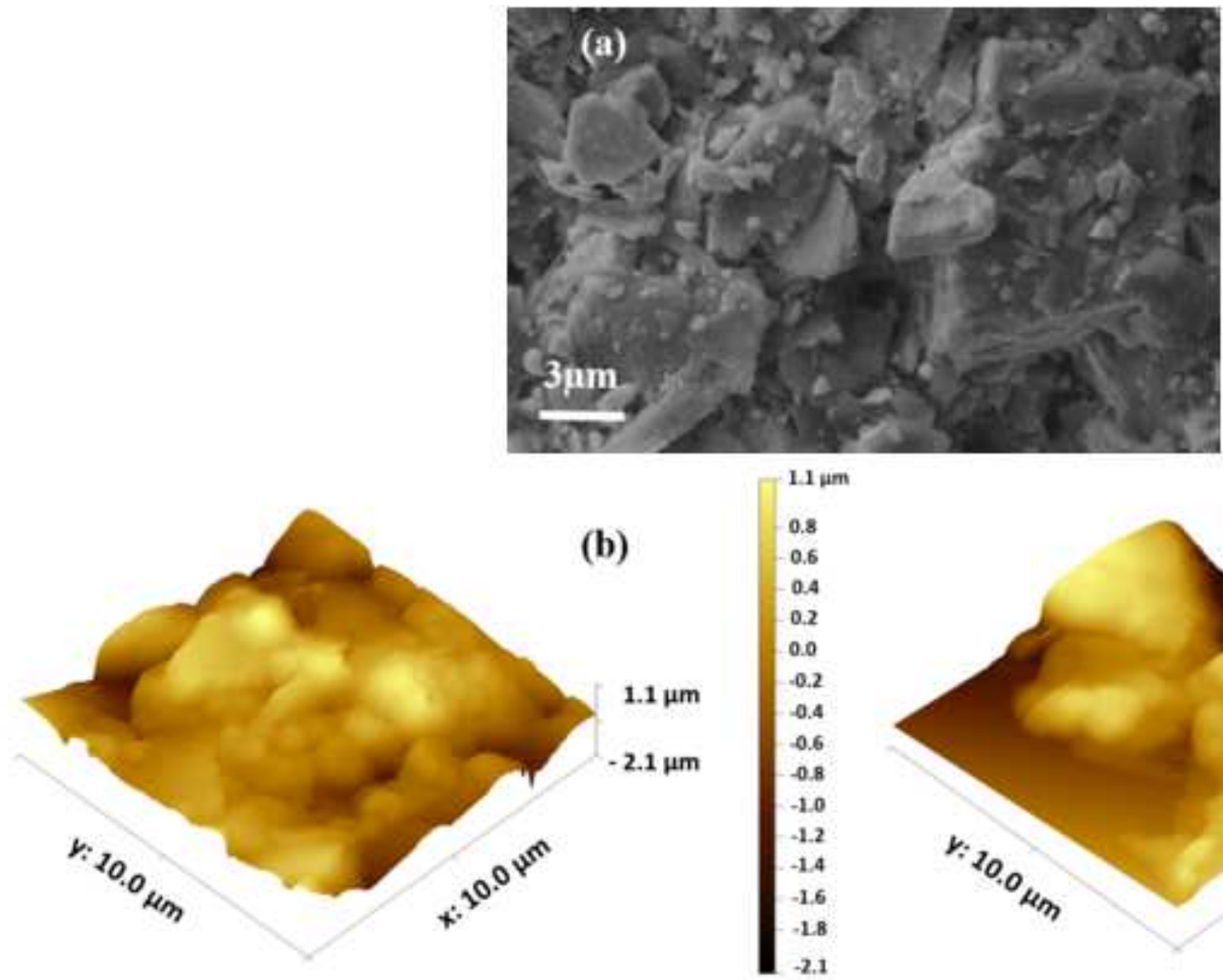

$1.1 \mu \mathrm{m}$
0.8
0.6
-0.4
0.2
0.0
-0.2
-0.4
-0.6
-0.8
-1.0
-1.2
-1.4
-1.6
-1.8
-2.1

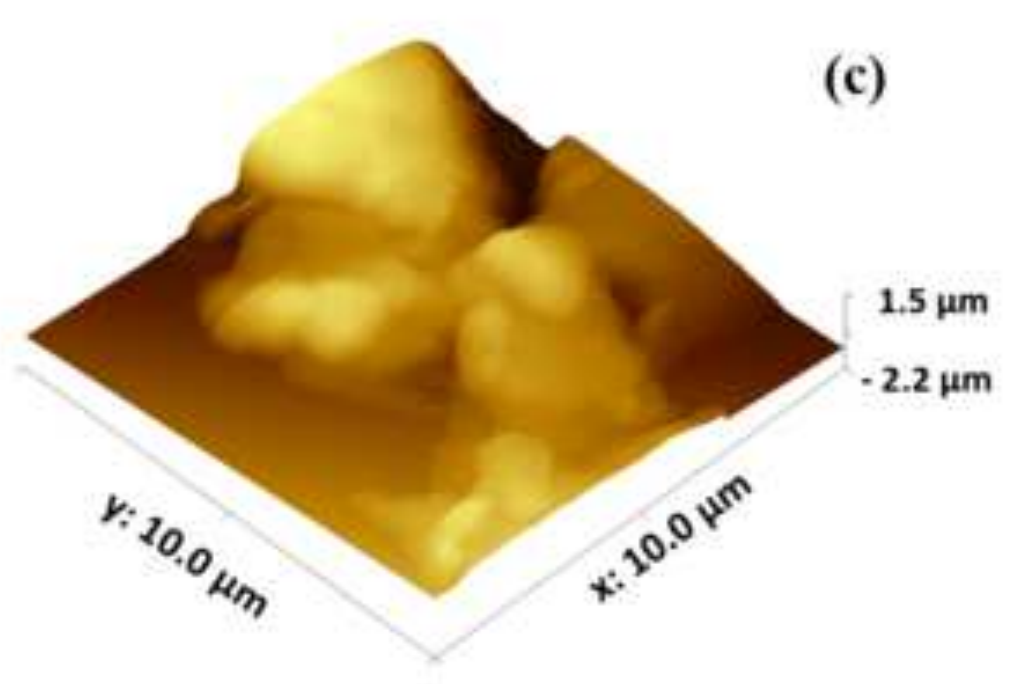

$1.5 \mu \mathrm{m}$
1.2
-1.0
0.8
0.6
0.4
0.2
0.0
-0.2
-0.4
-0.6
-0.8
-1.0
-1.2
-1.4
-1.6
-1.8
-2.0
-2.2



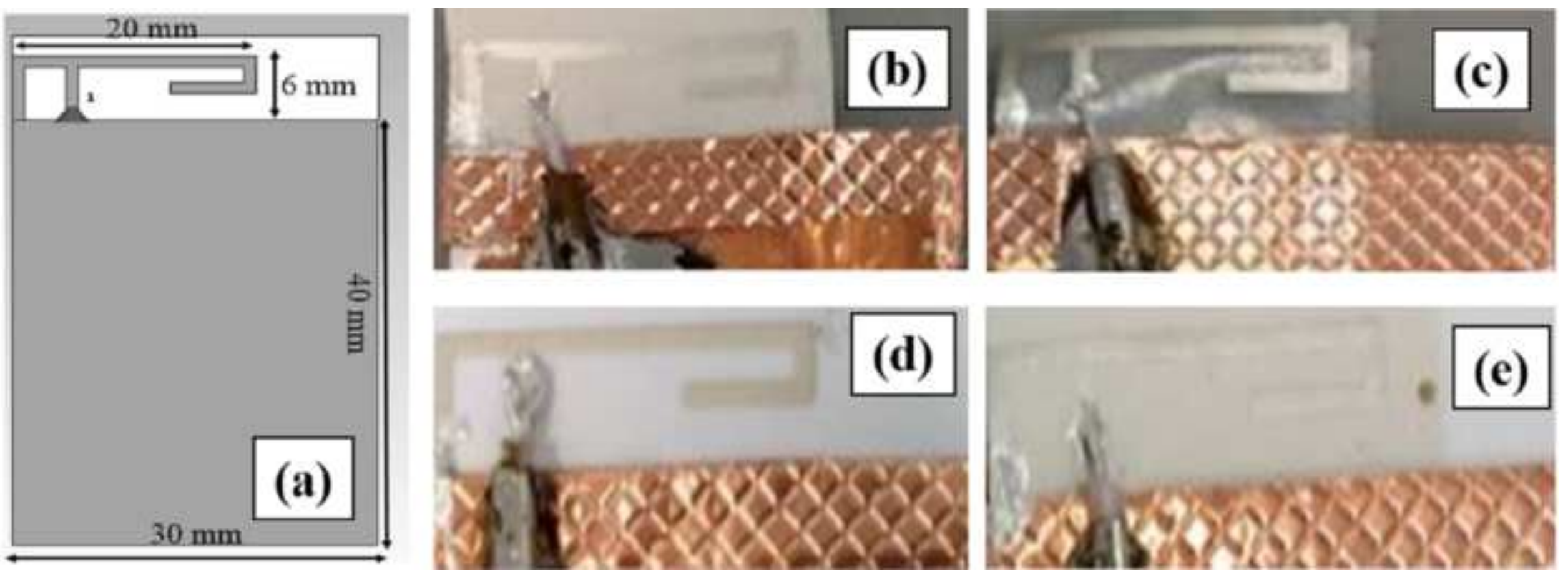

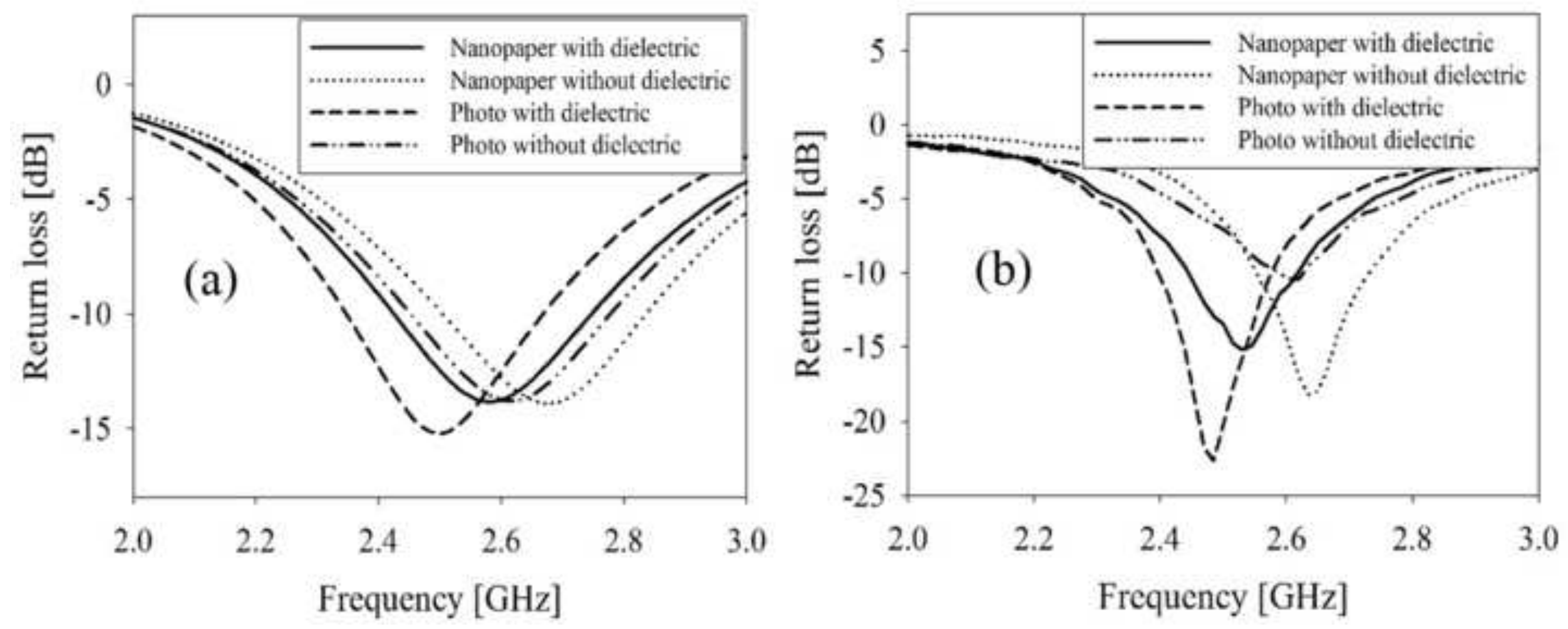

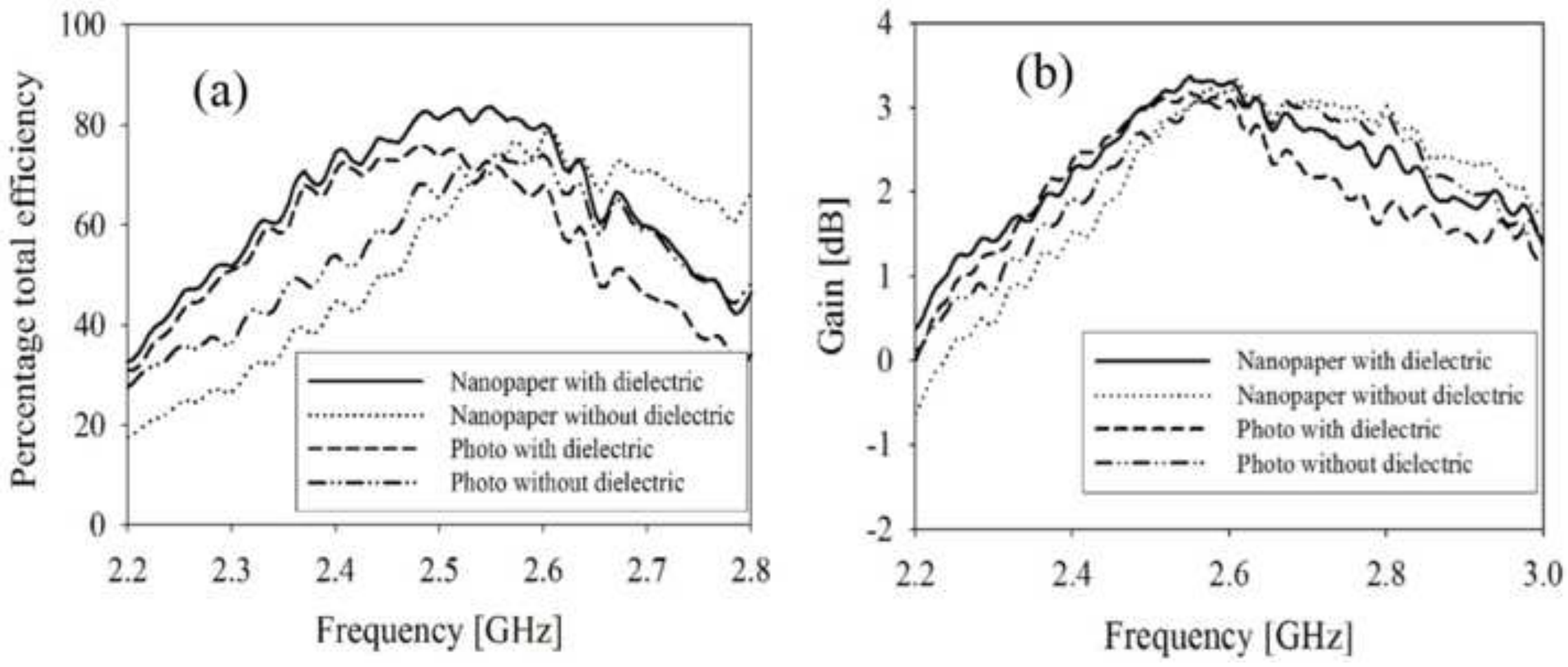


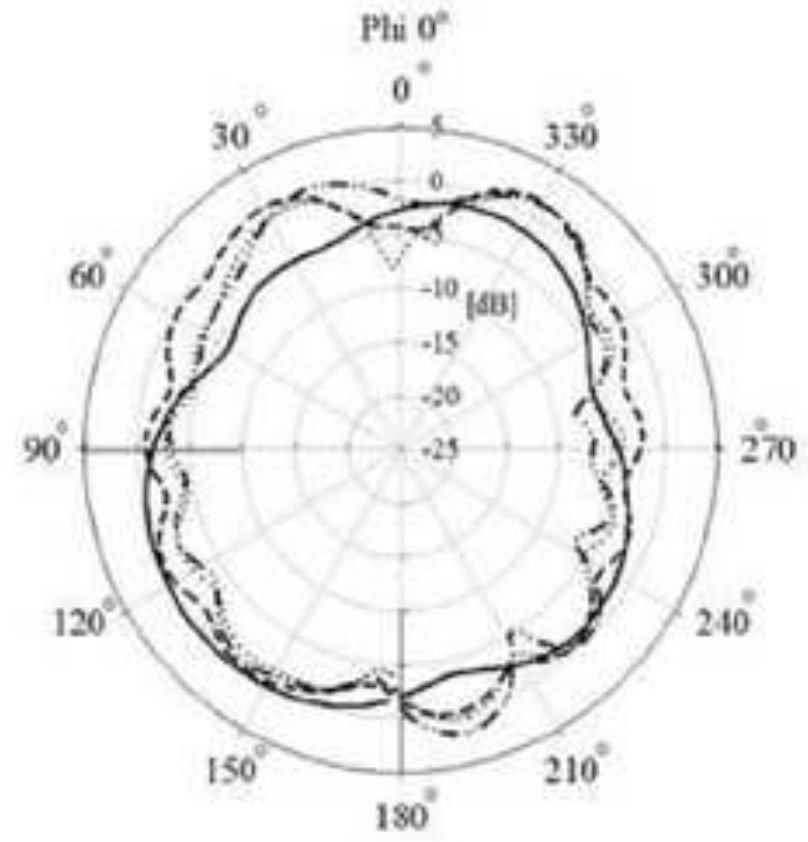

(a) XZ plane

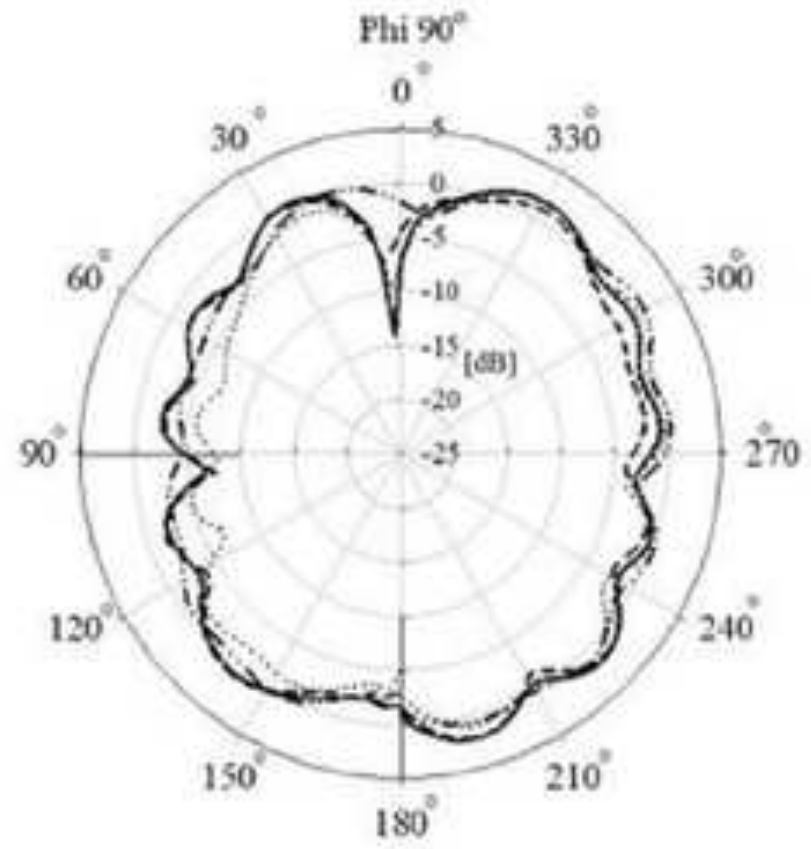

(b) YZ plane
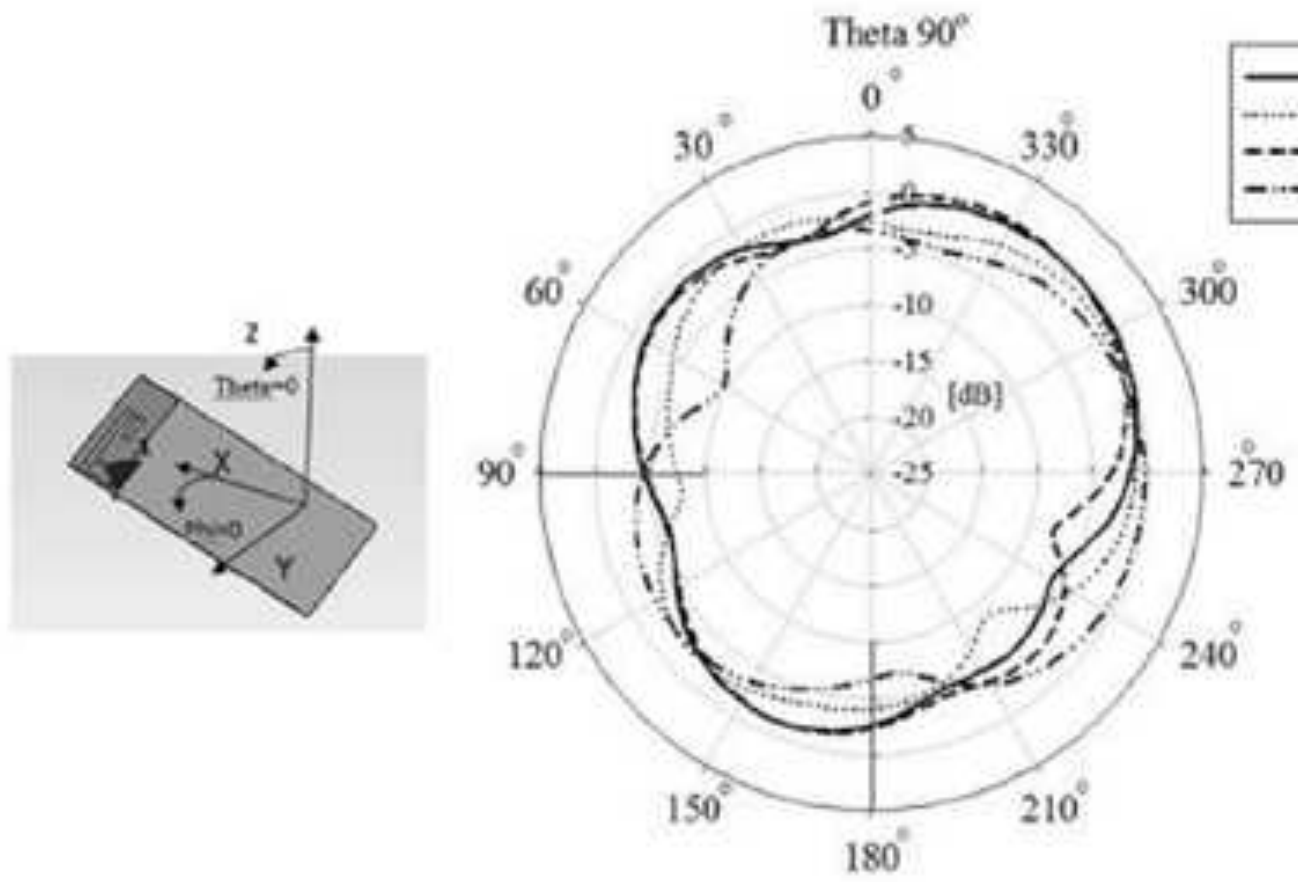

(c) XY plane 
Table I. Measured $\varepsilon r$ and tanঠ with and without dielectric ink at

\begin{tabular}{|l|c|c|c|}
\hline Sample name & Thickness $(\mathbf{m m})$ & $\begin{array}{l}\text { Relative permittivity } \\
\left(\varepsilon_{r}\right)\end{array}$ & $\begin{array}{l}\text { Loss tangent } \\
(\tan \delta)\end{array}$ \\
\hline Nanopaper & 0.03 & 4.70 & 0.12 \\
\hline $\begin{array}{l}\text { Nanopaper with dielectric } \\
\text { layer }\end{array}$ & 0.09 & 3.57 & 0.04 \\
\hline Photo & 0.20 & 3.12 & 0.09 \\
\hline Photo with dielectric layer & 0.28 & 2.61 & 0.05 \\
\hline
\end{tabular}

